\title{
Cortisol levels in pregnancy as a psychobiological predictor for birth weight
}

\author{
Margarete I. Bolten • Harald Wurmser • \\ Angelika Buske-Kirschbaum • Mechthild Papoušek • \\ Karl-Martin Pirke • Dirk Hellhammer
}

Received: 1 February 2010 /Accepted: 9 September 2010 /Published online: 25 September 2010

(C) Springer-Verlag 2010

\begin{abstract}
Antenatal maternal stress is thought to negatively affect fetal development, birth outcomes, and infant's development. Glucocorticoids are suggested to be a common link between prenatal stressors and infant's health. However, data on these mechanisms are rare and sometimes conflicting. The objective of this study was to examine the effects of maternal distress during pregnancy on fetal development and birth weight in humans prospectively. This study focuses on cortisol as one mediating the mechanism of the association between maternal distress and birth outcomes. Pregnancy-related and general distress was measured in 81 women with uncomplicated, singleton pregnancies. The rise of salivary cortisol on awakening
\end{abstract}

M. I. Bolten $(\bowtie)$

Institute for Psychology,

Department of Clinical Child and Adolescent Psychology,

University of Basel,

Missionsstrasse 64a,

4055 Basel, Switzerland

e-mail: margarete.bolten@unibas.ch

H. Wurmser $\cdot$ M. Papoušek

Child Care Centre, Ludwig-Maximilians-University Munich,

Heiglhofstraße 63,

81377 Munich, Germany

A. Buske-Kirschbaum

Institute of Psychology,

Department of Biological Psychology Technical,

University of Dresden,

Zellescher Weg 17,

01062 Dresden, Germany

K.-M. Pirke • D. Hellhammer

Institute for Psychology, Department of Psychobiology,

University of Trier,

Johanniterufer 15,

54290 Trier, Germany
(CAR) was assessed in weeks $13-18$ and 35-37 postmenstrual age of pregnancy. Mothers completed a structured interview, the perceived stress scale, a widely used psychological instrument that provided a global measure of perceived stress, as well as the Prenatal Distress Questionnaire, a self-report questionnaire designed to assess worries and anxiety in pregnancy. Pre-, peri-, and postnatal medical risk factors as well as birth characteristics were extracted from medical records routinely kept by the attending obstetricians. Hierarchical multiple regressions indicate that maternal cortisol levels explained $19.8 \%$ of the variance in birth weight and $9 \%$ of the variance in body length at birth, even after controlling for gestational age, parity, prepregnancy BMI, smoking, and infant's sex. Newborns of mothers with higher cortisol levels in pregnancy had lower birth weights and were shorter at birth. An ANCOVA for repeated measures indicated that, after controlling for covariates, pregnancy-related as well as general distress in pregnancy did not influence cortisol levels after awakening (area under the curve). No significant associations between perceived stress and anthrometric measures at birth were found. In conclusion, maternal cortisol levels in pregnancy influence intrauterine growth and may be a better predictor for birth outcome than perceived stress.

Keywords Prenatal stress · Pregnancy · Cortisol · Neuroendocrine $\cdot$ Fetal growth $\cdot$ Birth weight

\section{Introduction}

Low birth weight is still one of the greatest problems faced by perinatal medicine. Psychological distress in pregnancy plays a key role in this research field. Heightened emotional stress during pregnancy may lead to poor birth outcomes, as 
well as long-term physiological, emotional, or behavioral disturbances. Effects of prenatal environment on fetal and child development have been studied in animals and humans (e.g., for review, see Glover et al. 2010; Lupien et al. 2009; Mastorci et al. 2009; Merlot et al. 2008; O'Donnell et al. 2009; Viltart and Vanbesien-Mailliot 2007). While animal studies allow an experimental induction of stress, stressful life events or preexisting stress levels in subjects are associated with different outcome variables in human studies. For example, Maric et al. (2010) explored the impact of the Belgrad bombing in 1999 on the main obstetric characteristics of newborns. The authors found decreased birth weight in exposed mothers, but no specific relation between the trimester of stress exposure and birth weight. Lederman et al. (2004) found similar effects in women experiencing the World Trade Center (WTC) attack in their first trimester. Term infants born to women who were pregnant on September 11, 2001 and who were living within a 2-mile radius of the WTC showed significant decrements in birth weight and body length at birth. In accord with these results, Xiong et al. (2008) also reported three times higher rates of low birth weight in full-term infants when mothers were exposed to the Hurricane "Katrina." Besides these extremely negative life events, other authors have reported associations between maternal mental distress and lower birth weight. For instance, Oths et al. (2001) found lower birth weights for babies of women with high strain jobs. Antenatal maternal psychiatric disorders and antenatal distress were associated with lower birth weight and higher percentage of birth weight below the 10th percentile for gestational age (Maina et al. 2008). Comparably, Henrichs et al. (2010) showed that in late pregnancy, maternal symptoms of anxiety or depression were related to fetal size and lower birth weight.

Potential mechanisms related to prenatal stress exposure and offspring outcome are still not well explored in humans. Much of our understanding comes from animal models and hence cannot be transferred directly to humans. Wadhwa (2005) suggests that prenatal stress effects are mediated, in part, via maternal-placental-fetal neuroendocrine mechanisms, with placental corticotropin-releasing hormone (CRH) and glucocorticoids playing a central role. He proposes three possible mechanisms of how signals of maternal stress may reach the fetus: trans-placental transport of maternal hormones, reduction in intrauterine blood flow, and stress-induced suppression of maternal immune functions (compare also Bellinger et al. 2008; Cottrell and Seckl 2009; Maccari et al. 2003; Merlot et al. 2008; Welberg and Seckl 2001). Glucocorticoids seem to be the one common link between prenatal stressors, intrauterine growth, and birth weight (Seckl and Meaney 2004; Sloboda et al. 2000). Of maternal plasma cortisol, 10-20\%, may cross the placenta and double amniotic fluid cortisol concentration (maternal/fetal ratio, 11: 4) (Gitau et al. 1998). Glover et al. (2009) report comparable results. They found correlations of $r=0.32$ between amniotic fluid and maternal plasma cortisol. Reports on the association between prenatal stress and cortisol levels in human pregnancy are sometimes conflicting. While some studies report increased cortisol levels when mothers experienced higher levels of stress (Obel et al. 2005) or anxiety (Glover et al. 2009; Sarkar et al. 2008), others found no such findings (Figueiredo and Costa 2009; Huizink et al. 2003). Some issues in assessing the associations between stress during pregnancy and offspring outcomes are considered important: Associations may depend on the population under study, different mechanisms are likely to operate at different stages of pregnancy (Van den Bergh et al. 2005), and the conceptualization and psychometric measurement of stress is important. Therefore, one possible explanation for the inconsistencies mentioned above may be the varying definitions and conceptualizations of prenatal stress across studies. The systematic review by Nast et al. (submitted) identified 45 different instruments assessing prenatal distress, which could be categorized in five categories: mood, anxiety, general stress (general perceived stress, daily hassles, and specific chronic socio-environmental stressors), life events, and general health.

Given the prior findings linking severe distress with low birthweight and the conflicting results with regard to the association between perceived prenatal stress, cortisol levels, and birth, it is a main interest of the present study to illuminate these associations in a more normalized sample with moderate stress levels. The main objective of our study was to investigate the relationship between prenatal distress of the mother and birth outcome, mediated by maternal cortisol levels. Based on the thoughts outlined above, we expected maternal prenatal distress and the cortisol awakening response during pregnancy to be significantly correlated with birth outcome measures (birth weight, body length, and head circumference).

\section{Methods}

Participants and recruitment

A total of 94 women were recruited as a part of a prospective study on child development undertaken at the University of Trier. Subjects were enrolled in collaboration with local obstetricians/gynecologists in private practice, clinic departments of gynecology and obstetrics, and information centers for pregnant women, and through advertisements in local newspapers. Women that met inclusion criteria (age of 16 years or older and fluency in 
German) were contacted by phone and briefly informed about the study's research protocol. Exclusion criteria were (a) severe medical complications (acute or chronic physical diseases, such as gestational diabetes, metabolic diseases, hypertension, and thyroid hyper function), (b) signs of fetal malformation, (c) multi-fetal pregnancies, and (d) psychiatric problems. One woman was excluded due to being pregnant with twins, six women declined further participation after the first contact, five women lack cortisol measurements at both assessment points, and one woman was excluded due to extremely high cortisol levels. Consequently, data from $81(86.2 \%)$ women were available for the present study. Comparisons between the selected sample of 81 and the 13 excluded women indicated no significant differences between the two groups on demographic, biological, or psychological variables. Eleven women joined the study after week 20 of gestation and were therefore excluded from analyses of early pregnancy cortisol. Women from the remaining early pregnancy subsample $(n=70)$ were at $15.26(\mathrm{SD}=3.18)$ weeks of gestation at first assessment. Six women had to be excluded from the late pregnancy assessment due to missing cortisol values resulting in a subsample of $n=75$.

All women were paid a compensation of 200 euros for participation in the initial project. The study protocol was approved by the Ethical Committee of the University of Trier and is consistent with the revised Helsinki Declaration of 1975 .

\section{Measurements}

\section{Demographic and medical characteristics}

Structured interviews were conducted by trained research assistants and assessed sociodemographic (e.g., age, education, occupation, income, and marital status) and medical information. To screen the use of tobacco and alcohol in pregnancy, participants were asked to recall the amount and frequency of cigarettes smoked per day and alcoholic drinks per week. The use of tobacco and alcohol were coded dichotomously as smoking of one or more cigarettes per day and drinking of more than one alcoholic beverage per week. Pre-, peri-, and postnatal complications as well birth characteristics, including anthropometrical data, and gestational age were collected from medical records. Pregnancies were dated using the last menstrual period or sonographic measurements if dates were uncertain.

\section{Prenatal distress}

PSS The perceived stress scale (PSS) (Cohen et al. 1983) is the most widely used psychological instrument for measuring the perception of stress and is designed to provide a global measure of context free stress. This 14-item scale assesses the frequency of experiencing a situation as unpredictable, uncontrollable, or overloading. Participants indicate on a five-point scale from "never" to "very often" how frequently they experienced subjective affective and cognitive stress reactions, subjective effectiveness of and confidence about coping efforts, and subjective controllability of potential stressors during the past month. Internal consistency ranges between $\alpha=0.84$ and 0.86 . The PSS was administered at both assessment points.

$P D Q$ The prenatal distress questionnaire (PDQ) (Yali and Lobel 1999) is a 12-item self-report questionnaire designed to assess worries and anxiety of the pregnant mother regarding pregnancy and birth. The 12 items describe pregnancy-specific worries (e.g., medical complications, physical changes, birth, and health of the baby) that have to be rated on a five-point scale ranging from "not at all worried" to "extremely worried." Internal consistency for the English version is $\alpha=0.81$. We administered our own German translation at both assessment points of the study. Internal consistency of our German version was $\alpha=0.81$.

\section{Salivary cortisol}

Saliva samples were collected on two workdays at weeks 13-18 (early pregnancy) and at weeks 35-37 (late pregnancy) of gestation with the Salivette sampling device (Sarstedt, Nümbrecht, Germany) on awakening, and 30, 45, and $60 \mathrm{~min}$ after awakening. We asked participants to set an alarm clock at 7:00 a.m. and to collect the first sample immediately after awakening. It is well documented that cortisol levels in saliva reflect levels of the unbound steroid fraction in blood (Kirschbaum and Hellhammer 1994). Area under the curve (AUCg) of the cortisol awakening response was calculated using the trapezoid method with respect to ground (Pruessner et al. 2003). The AUCg is the most important measure of total hormonal secretion (Fekedulegn et al. 2007). This measure has been adopted most widely in studies involving repeated measures datasets and represents the total cortisol output and reflects the prominently high cortisol concentrations after awakening during pregnancy. The AUCg is more reliable than the area under the curve with respect to increase (Hellhammer et al. 2007).

Biochemical analysis

Samples were stored at $-20^{\circ} \mathrm{C}$ until being analyzed. On the day of testing, samples were thawed and centrifuged at $2,000 \times g$ at $10^{\circ} \mathrm{C}$ for $10 \mathrm{~min}$, and a clear sample was pipetted 
into appropriate test wells. All analyses were conducted under strict quality control. All samples from each subject were analyzed in the same assay batch to minimize variability using a commercial competitive luminescence immunoassay (LIA; IBL, Hamburg, Germany). Intra- and inter-assay variability was less than $10 \%$. The inter-assay variability was $3.9 \%$ for high and $7.1 \%$ for low concentrations. One out of ten samples was assayed in duplicate. The mean of the duplicate samples was used for statistical analyses.

\section{Data analysis}

Exploratory data analysis included examination of variables for missing data, normality, and outliers. Missing value analysis did not reveal any systematic patterns of missing data. Missing questionnaire items were replaced by the mean of the corresponding scale or subscale of the individual if applicable. Differences on demographic characteristics, salivary cortisol levels, and psychological stress measures between included and excluded study participants were tested by $t$ and $\chi^{2}$ tests. Differences in the cortisol awakening response between high and low stress groups were tested by repeated measures analysis of variance (ANOVA) using log transformed cortisol values. We entered the repeated factor data point (early and late pregnancy), the repeated factor cortisol $\left(t_{0}, t_{30}, t_{45}\right.$, and $\left.t_{60}\right)$, and the two stress scores (high and low levels PDQ and PSS by median split) as the between factors into the models. Since sphericity requirements were not met, results were Huynh-Feldt-corrected (uncorrected $d f$ 's reported). To assess the impact of free cortisol on birth outcome measures (birth weight, body length, and head circumference), three hierarchical regression analysis were performed. Six covariates (maternal age, parity, pre-pregnancy body mass index, gestational age, number of smoked cigarettes, and infant's sex) were entered in the first step. In a second step, we included the two stress scores (PDQ and PSS) to evaluate the effect of perceived prenatal distress on birth outcome variables. Cortisol levels after awakening (AUCg) measured in early and late pregnancy were included in the last step. For the multiple regression analysis, variables without normal distribution were log-transformed. To reduce the effect of multicollinearity on regression coefficients, we controlled for the tolerance coefficient. Tolerance coefficients of covariates and predictors were between 0.47 and 0.86 , indicating that between $47 \%$ and $86 \%$ of the explained variance is independent of the variance explained by other variables. The level of significance for all analyses was set at $\alpha=0.05$. The alpha of multiple independent $t$ and $\chi^{2}$ tests was adjusted according to Bonferroni. Statistical analyses were carried out using SPSS Version 16.0 for Mac OS X.

\section{Results}

Descriptive analyses

The sample mainly consisted of well-educated Caucasian women $(94.2 \%)$ with a high proportion in a medium (43.4\%) to high family income (14.5\%) bracket. Participants mean age was 31.4 years (SD 5.3). The following education levels were observed: $5.7 \%$ had less than 8 years of schooling, $57.1 \%$ had 10 to 12 years schooling, and $37.2 \%$ had a university degree. Eight $(9.9 \%)$ women continued smoking, but there was no self-reported alcohol or substance abuse in the sample.

Fourteen pregnancies were terminated by cesarean section, with three cesarean sections before week 37 (weeks 35 and 33). Two of the cesarean sections were elective; none of the vaginal deliveries were induced. Gestation was slightly shorter in women with initiated deliveries $\left(T_{52}=2.07 ; p=\right.$ $0.06 ; 39.7$ weeks postmenstrual age (pma), SD 1.3 vs. 38.2 weeks pma, SD 2.5).

Table 1 shows the descriptive statistics of predictors, covariates, and dependent variables. The mean pregnancyspecific distress score was 14.47 (SD 7.91) and covered the

Table 1 Descriptives of predictors, covariates and dependent variables $(N=81)$

\begin{tabular}{|c|c|c|}
\hline Variable & $M / \%$ & SD \\
\hline PDQ score & 14.5 & 7.9 \\
\hline PSS score & 24.0 & 9.7 \\
\hline Maternal age in years & 31.4 & 5.3 \\
\hline \multicolumn{3}{|l|}{ Parity } \\
\hline Primipara & $37.2 \%$ & \\
\hline Multipara & $62.8 \%$ & \\
\hline Pre-pregnancy BMI & 23.4 & 3.7 \\
\hline \multicolumn{3}{|l|}{ Smoking } \\
\hline Yes & $9.9 \%$ & \\
\hline No & $90.1 \%$ & \\
\hline Cigarettes per day & 1.4 & 4.7 \\
\hline \multicolumn{3}{|l|}{ Sex } \\
\hline Male & $44.9 \%$ & \\
\hline Female & $55.1 \%$ & \\
\hline Gestational age $^{\mathrm{a}}$ & 39.3 & 1.8 \\
\hline AUCg CAR early (13-18 weeks pma) & $1,653.0$ & 456.3 \\
\hline AUCg CAR late (35-37 weeks pma) & $1,950.8$ & 530.8 \\
\hline Birth weight ${ }^{b}$ & 3,421 & 551.5 \\
\hline Body length & 52.3 & 3.1 \\
\hline Head circumference $^{\mathrm{b}}$ & 34.9 & 1.8 \\
\hline
\end{tabular}

$P D Q$ prenatal distress questionnaire, $P S S$ perceived stress scale, $A U C g$ area under the curve, $C A R$ cortisol awakening rise, pma postmenstrual age, $B M I$ body mass index

${ }^{\mathrm{a}} 9.4 \%$ of sample $<37$ weeks

b $3.8 \%$ of sample $<2.500 \mathrm{~g}$ 
range observed by Yali and Lobel (1999a) as well as by Lobel et al. (2000). The mean PSS score was 24.04 (SD 9.71). Cortisol levels in early (13-18 weeks pma) and late pregnancy (35-37 weeks pma) were significantly correlated $(r=0.59 ; p<0.001)$. Babies were born on average after 39.3 (SD 1.8) weeks pma, with an average birth weight of $3421.13 \mathrm{~g}$ (SD 551), body length of $52.3 \mathrm{~cm}$ (SD 3.1), and head circumference of $34.9 \mathrm{~cm}$ (SD 1.8). All newborns had 5-min Apgar scores of 9 or 10. No baby was malformed.

Effects of maternal distress on free cortisol after awakening

Two three-way ANOVAs with repeated measures showed that pregnancy-specific as well as non-specific distress had no influence on cortisol levels. After awakening, cortisol levels showed the expected rise $\left(F=5.09, p<0.01, \omega^{2}=\right.$ 0.12 ). There was a significant increase in free salivary cortisol levels from early to late pregnancy $(F=19.85, p<$ $0.001, \omega^{2}=0.35$ ). Contrary to our expectations, maternal distress was not associated with cortisol secretion after awakening (AUCg) (PDQ: $F=1.40$, n.s., $1-\beta=0.92$, PSS: $F=0.62$, n.s., $1-\beta=0.97$ ). In our sample, we did not observe higher cortisol levels in women who reported more pregnancy specific nor unspecific stress.

Association between cortisol after awakening and infant's birth outcome measures

Results of the multiple hierarchical regression analysis are displayed in Table 2. Data revealed significant associations between maternal cortisol secretion after awakening and birth outcome measures after controlling for maternal age, pre-pregnancy BMI, parity, smoking, infant's sex, and gestational age. The proportion of variance explained by cortisol was $19.8 \%$ for birth weight and $9 \%$ for body length at birth. While free salivary cortisol after awakening in early as well as in late pregnancy predicted birth weight, only cortisol in late pregnancy was significantly associated with body length of the newborns. Cortisol after awakening did not explain a significant amount of variance in head circumference in our sample. Pregnancy-specific and unspecific stress was not associated to anthropometrical measures.

Concerning the covariates, gestational age predicted all anthropometrical measures. Infant's sex was significantly associated with birth weight, with boys weighing more than girls. Maternal age, parity, pre-pregnancy BMI, and the number of cigarettes smoked per day did not explain a significant amount of variance in anthropometrical measures.

\section{Discussion}

In the present study, we attempted to evaluate whether perceived distress during pregnancy influences birth outcome measures and if maternal cortisol secretion after awakening mediates this relation in 70 healthy women. The cortisol rise after awakening is considered to be an indicator of basal hypothalamic-pituitary-adrenal (HPA) axis activ-
Table 2 Summary of hierarchical regression analysis predicting anthropometric measures at birth $(n=70)$

$A U C \mathrm{~g}$ area under the curve, $C A R$ cortisol awakening rise, $B M I$ body mass index

$* p<0.05 ; * * p<0.01 ; * * * p<$ 0.001

\begin{tabular}{lllc}
\hline & $\begin{array}{l}\text { Birth weight } \\
\text { Cortisol levels after awakening }\end{array}$ & $\begin{array}{l}\text { Body length } \\
\text { Covariates and predictors }\end{array}$ & $\begin{array}{l}\text { Head circumference } \\
\beta\end{array}$ \\
\hline Step 1: Covariates & & & \\
Maternal age & 0.07 & 0.01 & -0.002 \\
Parity & 0.13 & 0.16 & 0.18 \\
Pre-pregnancy BMI & 0.21 & 0.21 & 0.04 \\
Number of cigarettes per day & -0.02 & -0.08 & 0.04 \\
Infant's sex & $-0.25^{*}$ & -0.20 & -0.18 \\
Gestational age & $0.55^{* * *}$ & $0.48^{* * *}$ & $0.48^{* * *}$ \\
$\Delta R^{2}$ for step 1 & $0.386^{* * *}$ & $0.318^{* *}$ & $0.298^{* *}$ \\
Step 2: Predictors & & & -0.30 \\
Prenatal distress questionnaire & -0.18 & -0.16 & 0.26 \\
Perceived stress scale & 0.04 & 0.05 & 0.052 \\
$\Delta R^{2}$ for step 2 & 0.022 & 0.014 & -0.07 \\
Step 3: Predictors & & & -0.22 \\
AUCg CAR early pregnancy & $-0.29^{*}$ & -0.08 & 0.055 \\
AUCg CAR late pregnancy & $-0.30^{* *}$ & $-0.28^{*}$ & $2.994^{* *}$ \\
$\Delta R^{2}$ for step 3 & $0.198^{* * *}$ & $0.090^{*}$ & \\
$F_{\text {Model }}$ & $6.915^{* * *}$ & $3.297^{* *}$ & \\
\hline
\end{tabular}


ity. Even though the $11 \beta$-HSD- 2 partly protects the fetus from maternal cortisol (Benediktsson et al. 1997), a small amount of cortisol is able to pass (Murphy et al. 1974). It seems that these glucocorticoids effect the intrauterine environment, which may affect the fetus (Seckl and Meaney 2004). However, the mechanisms in which prenatal stress affects fetal development and birth outcome measures are not yet well evaluated in humans. It is generally accepted that the HPA axis is a principal stress system. Therefore, hormones of the pituitary-adrenal system are suggested as the endocrine link between prenatal stress and birth outcome.

The major finding of the present study indicates that even after controlling for maternal age, parity, prepregnancy BMI, number of cigarettes smoked per day, infant's sex, and gestational age, maternal cortisol levels after awakening (AUCg) explained 19.8\% of the variance in birth weights and $9 \%$ of the variance in body length. Our findings are in line with findings from studies that have shown reductions in weight at birth after administration of several doses of glucocorticoids in animals and humans (Bloom et al. 2001; French et al. 1999). Elevated free circulating cortisol may pass through the placenta, slow fetal growth rate, and reduce birth weight of prenatally stressed offspring. Sloboda et al. (2000) could show that weight at birth of merino ewes was significantly reduced by $23 \%$ at 125 days and $19 \%$ at 146 days of gestation after exposure to betamethasone. Comparably, betamethasone exposure caused growth retardation and decreased body weights in lambs (Jobe et al. 2003; Jobe et al. 1998). Data suggest that higher levels of cortisol in pregnancy may be a risk factor for lower birth weight caused by the catabolic potency of the glucocorticoids.

Even though studies assessing associations between prenatal distress and offspring development often assume that high levels of stress cause high levels of cortisol, our results showed no relation between maternal distress in pregnancy and levels of free cortisol in saliva after awakening in pregnancy (AUCg). Consistent with these findings, we found no associations between perceived stress and any anthropometric measure at birth. These findings are in accord with other human studies that find no or only weak correlations between psychological measures in pregnancy and cortisol levels. For example, Wadhwa et al. (1996) found no significant association between life events stress and plasma cortisol. Comparably, Figueiredo and Costa (2009) found no significant psychosocial predictors for maternal cortisol levels 3 months before childbirth. Obel et al. (2005) reported inconsistent findings showing that women who reported pregnancy-specific stress due to pregnancy complications exhibited lower morning cortisol levels in early pregnancy, but subjects who experienced more life events and stress and were worried about existing pregnancy complications exhibited higher evening cortisol levels in late pregnancy. Shea et al. (2007) found lower baseline awakening cortisol levels in pregnant women with higher childhood trauma scores at week 20 of gestation.

Other research groups determined higher cortisol levels in pregnant women with anxiety or depression. Evans et al. (2008) found elevated cortisol levels during a laboratory stress test only in the comorbid group (anxiety and depression), but subjects with only one diagnosis did not differ from the control group. Similarly, Field and Diego (2008) and Field et al. (2006) reported positive correlations between anxiety, depression, and maternal cortisol levels in pregnancy. These inconsistencies reported above may be caused by differences in stress operationalization across studies (life events, pregnancy-related distress, childhood trauma, anxiety, or depression). While animal studies allow an experimental induction of chronic stress across randomized groups, human studies are limited to quasiexperimental study designs with subjects varying in levels of preexisting stress, experiencing of stressful events, or in the experimental manipulation of short-term stress effects.

Another explanation why we did not find associations between prenatal stress and cortisol levels after awakening may be that morning values are at their peak and it would take remarkably high stress levels to raise the already elevated levels. Morning levels of salivary cortisol significantly increased from early to late pregnancy in our study. With progressing pregnancy, plasma levels of $\mathrm{CRH}$ increase by the rising CHR production in intrauterine tissues (Challis et al. 2000). As a result, maternal pituitary adrenocorticotrophic hormone (ACTH) secretion and plasma ACTH levels, as well as total and free cortisol levels, rise (Allolio et al. 1990). Although we did not found a significant association between maternal distress and cortisol secretion after awakening, it cannot be ruled out that extreme levels of psychosocial stress may result in enhanced cortisol levels. In contrast to other studies, we measured cortisol levels after awakening and used the area under the curve with respect to ground (AUCg) as predictor. Although the cortisol rise after awakening (CAR) is a frequently applied measure of pituitary-adrenal activity, which has high intra-individual stability (Hucklebridge et al. 2005) and can be easily be assessed in saliva samples collected by the subject under real life conditions, this index has a number of weaknesses. The CAR cannot be observed in $25 \%$ of healthy adults (Wust et al. 2000). The CAR is relatively distinct from the diurnal variations in HPA axis activity and is rather an additional phenomenon reflecting specific processes associated with awakening (Wilhelm et al. 2007). Therefore, it could be important for future research to study the associations between perceived stress, diurnal or evening cortisol levels, and birth weight. It should also be considered that the HPA axis is a highly adaptive system, 
which is characterized by marked intra- and interindividual variability. The salivary cortisol secretion might be influenced by many other factors. A recent review (Hansen et al. 2008) provides an overview of variations in saliva cortisol levels in field studies. A multitude of studies within the last years examined interindividual variations with regard to cortisol levels. Variability can be both biological and methodological in origin. One major problem in field studies involving data collections by the participants themselves is the possible noncompliance to the sampling protocol, especially concerning the correct timing of sampling (Kudielka et al. 2003). Seasonal variations have been reported by Persson et al. (2008). Besides gender and age, life-style variables (e.g., sleep pattern, diet, medication, drug consumption, and physical activity) can also have an impact on cortisol secretion. For example, sleep duration and sleep quality as well as awakening seem to be potent stimuli to trigger cortisol release. Patients with insomnia are reported to display blunted salivary cortisol levels after awakening (Backhaus et al. 2004). Time of awakening (Federenko et al. 2004) and nocturnal awakening (Hucklebridge et al. 2000), what is found frequently in pregnant women, may also affect salivary cortisol levels. Food intake (Hershberger et al. 2004), caffeine (Lovallo et al. 2005), and moderate physical activity (Frey 1982) may be physiological challenges to the HPA axis. Although most pregnant women stay abstinent from alcohol and nicotine, both can have pronounced effects on the endocrine function. Cigarette smoking (Rohleder and Kirschbaum 2006) as well as moderate alcohol consumption (Kokavec et al. 2009) can affect cortisol levels. In humans, it is difficult to control for all confounding variables and to establish a causal link between prenatal stress and birth outcomes.

Another reason may be the small sample size and therefore little variance in stress levels. We were not able to compare extreme groups with regard to maternal distress. Maternal HPA axis would only be changed if a critical threshold of stress were exceeded.

Despite these limitations, the current data provide evidence that higher levels of cortisol after awakening, which is a stable marker of adrenal function, are associated with lower birth weight of the offspring. It can be ascertained that high cortisol levels in pregnancy may be a risk factor for low birth weight. Women's cortisol levels after awakening were not explained by perceived prenatal stress. Further studies are needed to explore if perceived stress during pregnancy influences cortisol levels using larger samples and repetitive cortisol measures during the day.

Acknowledgement This study was supported by a grant from the German Research foundation (PA 376/6-1, 6-2, and 6-3 and PI 143/71, 7-2, and 7-3). We thank Dr. Pathik Wadhwa and Dr. Kristen Lavallee for their comments on an earlier draft of this article.

\section{References}

Allolio B, Hoffmann J, Linton EA, Winkelmann W, Kusche M, Schulte HM (1990) Diurnal salivary cortisol patterns during pregnancy and after delivery: relationship to plasma corticotrophin-releasinghormone. Clin Endocrinol (Oxf) 33(2):279-289

Backhaus J, Junghanns K, Hohagen F (2004) Sleep disturbances are correlated with decreased morning awakening salivary cortisol. Psychoneuroendocrinology 29(9):1184-1191

Bellinger DL, Lubahn C, Lorton D (2008) Maternal and early life stress effects on immune function: relevance to immunotoxicology. J Immunotoxicol 5(4):419-444

Benediktsson R, Calder AA, Edwards CR, Seckl JR (1997) Placental 11 beta-hydroxysteroid dehydrogenase: a key regulator of fetal glucocorticoid exposure. Clin Endocrinol (Oxf) 46(2):161-166

Bloom SL, Sheffield JS, McIntire DD, Leveno KJ (2001) Antenatal dexamethasone and decreased birth weight. Obstet Gynecol 97 (4):485-490

Challis J, Sloboda D, Matthews S, Holloway A, Alfaidy N, Howe D et al (2000) Fetal hypothalamic-pituitary adrenal (HPA) development and activation as a determinant of the timing of birth, and of postnatal disease. Endocr Res 26(4):489-504

Cohen S, Kamarck T, Mermelstein R (1983) A global measure of perceived stress. J Health Soc Behav 24(4):385-396

Cottrell EC, Seckl JR (2009) Prenatal stress, glucocorticoids and the programming of adult disease. Front Behav Neurosci 3:19

Evans LM, Myers MM, Monk C (2008) Pregnant women's cortisol is elevated with anxiety and depression - but only when comorbid. Arch Womens Ment Health 11(3):239-248

Federenko I, Wust S, Hellhammer DH, Dechoux R, Kumsta R, Kirschbaum C (2004) Free cortisol awakening responses are influenced by awakening time. Psychoneuroendocrinology 29 (2): 174-184

Fekedulegn DB, Andrew ME, Burchfiel CM, Violanti JM, Hartley TA, Charles LE et al (2007) Area under the curve and other summary indicators of repeated waking cortisol measurements. Psychosom Med 69(7):651-659

Field T, Diego M (2008) Cortisol: the culprit prenatal stress variable. Int J Neurosci 118(8):1543-5245

Field T, Hernandez-Reif M, Diego M, Schanberg S, Kuhn C (2006) Stability of mood states and biochemistry across pregnancy. Infant Behav Dev 29(2):262-267

Figueiredo B, Costa R (2009) Mother's stress, mood and emotional involvement with the infant: 3 months before and 3 months after childbirth. Arch Womens Ment Health 12(3):143-153

French NP, Hagan R, Evans SF, Godfrey M, Newnham JP (1999) Repeated antenatal corticosteroids: size at birth and subsequent development. Am J Obstet Gynecol 180(1 Pt 1):114-121

Frey H (1982) The endocrine response to physical activity. Scand J Soc Med Suppl 29:71-75

Gitau R, Cameron A, Fisk NM, Glover V (1998) Fetal exposure to maternal cortisol. Lancet 352(9129):707-708

Glover V, Bergman K, Sarkar P, O’Connor TG (2009) Association between maternal and amniotic fluid cortisol is moderated by maternal anxiety. Psychoneuroendocrinology 34(3):430-435

Glover V, O'Connor TG, O'Donnell K (2010) Prenatal stress and the programming of the HPA axis. Neurosci Biobehav Rev 35:17-22

Hansen AM, Garde AH, Persson R (2008) Sources of biological and methodological variation in salivary cortisol and their impact on measurement among healthy adults: a review. Scand J Clin Lab Invest 68(6):448-458

Hellhammer J, Fries E, Schweisthal OW, Schlotz W, Stone AA, Hagemann D (2007) Several daily measurements are necessary to reliably assess the cortisol rise after awakening: state- and trait components. Psychoneuroendocrinology 32(1):80-86 
Henrichs J, Schenk JJ, Roza SJ, van den Berg MP, Schmidt HG, Steegers EA et al (2010) Maternal psychological distress and fetal growth trajectories: the Generation R Study. Psychol Med 40:633-643

Hershberger AM, McCammon MR, Garry JP, Mahar MT, Hickner RC (2004) Responses of lipolysis and salivary cortisol to food intake and physical activity in lean and obese children. J Clin Endocrinol Metab 89(9):4701-4707

Hucklebridge F, Lambert S, Clow A, Warburton DM, Evans PD, Sherwood N (2000) Modulation of secretory immunoglobulin A in saliva; response to manipulation of mood. Biol Psychol 53 (1):25-35

Hucklebridge F, Hussain T, Evans P, Clow A (2005) The diurnal patterns of the adrenal steroids cortisol and dehydroepiandrosterone (DHEA) in relation to awakening. Psychoneuroendocrinology 30(1):51-57

Huizink AC, Robles de Medina PG, Mulder EJ, Visser GH, Buitelaar JK (2003) Stress during pregnancy is associated with developmental outcome in infancy. J Child Psychol Psychiatry 44 (6):810-818

Jobe AH, Wada N, Berry LM, Ikegami M, Ervin MG (1998) Single and repetitive maternal glucocorticoid exposures reduce fetal growth in sheep. Am J Obstet Gynecol 178(5):880-885

Jobe AH, Newnham JP, Moss TJ, Ikegami M (2003) Differential effects of maternal betamethasone and cortisol on lung maturation and growth in fetal sheep. Am J Obstet Gynecol 188(1):2228

Kirschbaum C, Hellhammer DH (1994) Salivary cortisol in psychoneuroendocrine research: recent developments and applications. Psychoneuroendocrinology 19(4):313-333

Kokavec A, Lindner AJ, Ryan JE, Crowe SF (2009) Ingesting alcohol prior to food can alter the activity of the hypothalamic-pituitaryadrenal axis. Pharmacol Biochem Behav 93(2):170-176

Kudielka BM, Broderick JE, Kirschbaum C (2003) Compliance with saliva sampling protocols: electronic monitoring reveals invalid cortisol daytime profiles in noncompliant subjects. Psychosom Med 65(2):313-319

Lederman SA, Rauh V, Weiss L, Stein JL, Hoepner LA, Becker M et al (2004) The effects of the World Trade Center event on birth outcomes among term deliveries at three lower Manhattan hospitals. Environ Health Perspect 112(17):1772-1778

Lobel M, DeVincent CJ, Kaminer A, Meyer BA (2000) The impact of prenatal maternal stress and optimistic disposition on birth outcomes in medically high-risk women. Health Psychol 19(6):544-553

Lovallo WR, Whitsett TL, al'Absi M, Sung BH, Vincent AS, Wilson MF (2005) Caffeine stimulation of cortisol secretion across the waking hours in relation to caffeine intake levels. Psychosom Med 67(5):734-739

Lupien SJ, McEwen BS, Gunnar MR, Heim C (2009) Effects of stress throughout the lifespan on the brain, behaviour and cognition. Nat Rev Neurosci 10(6):434-445

Maccari S, Darnaudery M, Morley-Fletcher S, Zuena AR, Cinque C, Van Reeth O (2003) Prenatal stress and long-term consequences: implications of glucocorticoid hormones. Neurosci Biobehav Rev 27(1-2):119-127

Maina G, Saracco P, Giolito MR, Danelon D, Bogetto F, Todros T (2008) Impact of maternal psychological distress on fetal weight, prematurity and intrauterine growth retardation. J Affect Disord 111(2-3):214-220

Maric NP, Dunjic B, Stojiljkovic DJ, Britvic D, Jasovic-Gasic M (2010) Prenatal stress during the 1999 bombing associated with lower birth weight - a study of 3, 815 births from Belgrade. Arch Womens Ment Health 13:83-89

Mastorci F, Vicentini M, Viltart O, Manghi M, Graiani G, Quaini F et al (2009) Long-term effects of prenatal stress: changes in adult cardiovascular regulation and sensitivity to stress. Neurosci Biobehav Rev 33(2):191-203

Merlot E, Couret D, Otten W (2008) Prenatal stress, fetal imprinting and immunity. Brain Behav Immun 22(1):42-51

Murphy BE, Clark SJ, Donald IR, Pinsky M, Vedady D (1974) Conversion of maternal cortisol to cortisone during placental transfer to the human fetus. Am J Obstet Gynecol 118(4):538 541

Obel C, Hedegaard M, Henriksen TB, Secher NJ, Olsen J, Levine S (2005) Stress and salivary cortisol during pregnancy. Psychoneuroendocrinology 30(7):647-656

O'Donnell K, O'Connor TG, Glover V (2009) Prenatal stress and neurodevelopment of the child: focus on the HPA axis and role of the placenta. Dev Neurosci 31(4):285-292

Oths KS, Dunn LL, Palmer NS (2001) A prospective study of psychosocial job strain and birth outcomes. Epidemiology 12 (6):744-746

Persson R, Garde AH, Hansen AM, Osterberg K, Larsson B, Orbaek P et al (2008) Seasonal variation in human salivary cortisol concentration. Chronobiol Int 25(6):923-937

Pruessner JC, Kirschbaum C, Meinlschmid G, Hellhammer DH (2003) Two formulas for computation of the area under the curve represent measures of total hormone concentration versus time-dependent change. Psychoneuroendocrinology 28(7):916931

Rohleder N, Kirschbaum C (2006) The hypothalamic-pituitaryadrenal (HPA) axis in habitual smokers. Int J Psychophysiol 59 (3):236-243

Sarkar P, Bergman K, O’Connor TG, Glover V (2008) Maternal antenatal anxiety and amniotic fluid cortisol and testosterone: possible implications for foetal programming. J Neuroendocrinol 20(4):489-496

Seckl JR, Meaney MJ (2004) Glucocorticoid programming. Ann NY Acad Sci 1032:63-84

Shea AK, Streiner DL, Fleming A, Kamath MV, Broad K, Steiner M (2007) The effect of depression, anxiety and early life trauma on the cortisol awakening response during pregnancy: preliminary results. Psychoneuroendocrinology 32(8-10):1013-1020

Sloboda DM, Newnham JP, Challis JR (2000) Effects of repeated maternal betamethasone administration on growth and hypothalamic-pituitary-adrenal function of the ovine fetus at term. J Endocrinol 165(1):79-91

Van den Bergh BR, Mulder EJ, Mennes M, Glover V (2005) Antenatal maternal anxiety and stress and the neurobehavioural development of the fetus and child: links and possible mechanisms. A review. Neurosci Biobehav Rev 29(2):237-258

Viltart O, Vanbesien-Mailliot CC (2007) Impact of prenatal stress on neuroendocrine programming. SciWorld J 7:1493-1537

Wadhwa PD (2005) Psychoneuroendocrine processes in human pregnancy influence fetal development and health. Psychoneuroendocrinology 30(8):724-743

Wadhwa PD, Dunkel-Schetter C, Chicz-DeMet A, Porto M, Sandman CA (1996) Prenatal psychosocial factors and the neuroendocrine axis in human pregnancy. Psychosom Med 58 (5):432-446

Welberg LA, Seckl JR (2001) Prenatal stress, glucocorticoids and the programming of the brain. J Neuroendocrinol 13(2):113-128 
Wilhelm I, Born J, Kudielka BM, Schlotz W, Wust S (2007) Is the cortisol awakening rise a response to awakening? Psychoneuroendocrinology 32(4):358-366

Wust S, Wolf J, Hellhammer DH, Federenko I, Schommer N, Kirschbaum C (2000) The cortisol awakening response-normal values and confounds. Noise Health 2(7):79-88
Xiong X, Harville EW, Mattison DR, Elkind-Hirsch K, Pridjian G, Buekens P (2008) Exposure to Hurricane Katrina, post-traumatic stress disorder and birth outcomes. Am J Med Sci 336(2):111-115

Yali AM, Lobel M (1999) Coping and distress in pregnancy: an investigation of medically high risk women. J Psychosom Obstet Gynaecol 20(1):39-52 